\title{
EDITORIAL
}

\section{Application of Endoscopic Ultrasonography in the Diagnosis and Treatment of Lower Gastrointestinal Disease}

\author{
Eun Young Kim \\ Departments of Internal Medicine, Catholic University of Daegu School of Medicine, Daegu, Korea
}

\section{Article: The Clinical Usefulness of Endoscopic Ultrasound-Guided Fine Needle Aspiration and Biopsy for Rectal and Perirectal Lesions (Intest Res 2015;13:135-144)}

Endoscopic ultrasonography (EUS) currently plays an important role in the field of gastroenterology, particularly in the diagnosis and treatment of upper gastrointestinal (GI) and pancreatobiliary diseases. However, it remains underused in the lower GI diseases field, probably because of the lack of knowledge and experience of endosonographers.

The diagnostic value of EUS is well demonstrated in the local staging of rectal cancer, and it also shows benefits in the differential diagnosis of submucosal tumors of the lower GI tract; assessment of IBD with or without perirectal fistula; evaluation of perianal and perirectal inflammatory conditions; and evaluation of anal sphincters. ${ }^{1}$

In this issue of Intestinal Research, Soh et al. ${ }^{2}$ reported their experience of EUS-guided fine needle aspiration (FNA) and biopsy (FNB) for rectal and perirectal lesions. Previously, in 2011, the authors reported their outcome of EUS-FNA and trucut biopsy (TCB) for patients with rectal and perirectal lesions in another journal. ${ }^{3}$ They stated that from 12 EUS-FNA procedures performed in 11 patients with or without EUStrucut biopsy, a definite diagnosis was obtained in 10. The results obtained using EUS-guided sampling procedures avoided unnecessary or inappropriate surgery and allowed

Received March 31, 2015. Accepted March 31, 2015

Correspondence to Eun Young Kim, Department of Internal Medicine, Catholic University of Daegu School of Medicine, 33 Duryugongwon-ro 17gil, Nam-gu, Daegu 705-718, Korea. Tel: +82-53-650-4092, Fax: +82-53624-3281,E-mail:kimey@cu.ac.kr

Financial support: None. Conflict of interest: None. the timely administration of chemotherapy. In this article, they report new findings on the use of EUS-guided sampling in 30 patients with rectal and perirectal lesions which include the previously reported cases. Surprisingly enough, the diagnostic accuracy of EUS-FNA/FNB was only $67 \%(20 / 30)$ this time, which means that merely 10 accurate diagnoses were obtained after performing 19 additional consecutive procedures. Diagnostic accuracy of subepithelial tumor (SET) was only $50 \%(5 / 10)$ and that of non-SET was $75 \%$ $(15 / 20)$. They concluded that the size of the lesion was an important factor for the diagnostic accuracy of EUS-guided sampling of rectal SET. While the three SETs included in their first report measured $44 \mathrm{~mm}, 30 \mathrm{~mm}$, and $50 \mathrm{~mm}$ in size, among 10 SETs included in this paper, only 5 of them were larger than $20 \mathrm{~mm}$ in size. This factor may somewhat explain the discrepancies in EUS-guided sampling accuracy between their two reports.

Nevertheless, their diagnostic accuracy of EUS-guided sampling of rectal and perirectal lesions is lower than that of previous studies ranging 90-95.5\% ${ }^{4-6}$ The authors speculated that absence of on-site cytopathologist could be one of the reasons of the lower accuracy seen in this study. The result of EUS-guided sampling can also be influenced by the various factors such as experience of endosonographer, used instrument and technique. The authors did not mention on the practiced endosonographer(s) at all in their previous report, and in this article, they just disclosed the fact that five endosonographers performed the procedures, but did not provide additional information on them. In addition, a close

๑ Copyright 2015. Korean Association for the Study of Intestinal Diseases. All rights reserved.

This is an Open Access article distributed under the terms of the Creative Commons Attribution Non-Commercial License (http://creativecommons.org/licenses/by-nc/3.0)

which permits unrestricted non-commercial use, distribution, and reproduction in any medium, provided the original work is properly cited. 
look at their technique reveals that they did not apply negative pressure during FNA and $10 \mathrm{~mL}$ suction syringe was used only for EUS-FNB with ProCore needle. Applying negative pressure during EUS-FNA can help to obtain a larger sample but the harvest may contain more blood. So operator should decide whether to use negative pressure or not according to the characteristic of the target lesion. In applying negative pressure for EUS-guided sampling, the choice between slow-pull back of the stylet and syringe suction method is debatable, with some investigators suggesting that the slow-pull technique increases the diagnostic yield without blood contamination compared with syringe suction during EUS-FNA of pancreatic solid masses. ${ }^{7}$ Further study is needed concerning the technique of EUS-guided sampling of rectal and perirectal lesions to improve its outcome.

Soh et al. ${ }^{2}$ reported 2 cases of complication which were fever and pneumoperitoneum. Levy et al. ${ }^{8}$ reported adverse events in $20.5 \%$ of patients following lower GI EUS-FNA, of which, $5.6 \%$ were serious complications. Antibiotic prophylaxis is recommended to avoid EUS-FNA complications in cases where the needle is required to pass through the rectal wall or to be inserted into a cystic lesion.

Recently, EUS-guided therapeutic procedures such as EUS-guided pseudocyst drainage or biliary drainage have gained popularity in the pancreatobiliary field. EUS-guided pelvic abscess drainage is cautiously uprising as a safe and effective minimally invasive procedure. ${ }^{9}$ After accumulation of experience, EUS-guided drainage of pelvic collection will serve as an efficient treatment option in the treatment of complication of obstetric and colorectal surgery. Another therapeutic application of lower GI EUS is EUS-guided coil and glue injection on treating bleeding rectal varices. Investigators demonstrated that this procedure was safe and effective. ${ }^{10}$ Fiducial implantation for patients with localized rectal cancer receiving high-dose rate endorectal brachytherapy was reported as a new additional EUS-guided procedure. ${ }^{11}$

EUS is an effective tool that can significantly facilitate the diagnosis and treatment of lower GI and perirectal diseases. The usage of EUS in deep seated colonic lesion is limited due to the oblique viewing lens of currently used echoendoscope. However, when newly developed forward viewing echoendoscope is routinely employed in lower GI disease, the field of EUS including EUS-FNA and therapeutic utilization would be expanded widely before long.

\section{REFERENCES}

1. Zhu Q, Xu C, Hu D. Dose endoscopic ultrasonography impact diagnosis of intestinal diseases? Intest Res 2011;9:179-188.

2. Soh JS, Lee HS, Lee S, et al. The clinical usefulness of endoscopic ultrasound-guided fine needle aspiration and biopsy for rectal and perirectal lesions. Intest Res 2015;13:135-144.

3. Boo SJ, Byeon JS, Park do H, et al. EUS-guided fine needle aspiration and trucut needle biopsy for examination of rectal and perirectal lesions. Scand J Gastroenterol 2011;46:1510-1518.

4. Maleki Z, Erozan Y, Geddes S, Li QK. Endorectal ultrasoundguided fine-needle aspiration: a useful diagnostic tool for perirectal and intraluminal lesions. Acta Cytol 2013;57:9-18.

5. Sasaki Y, Niwa Y, Hirooka Y, et al. The use of endoscopic ultrasound-guided fine-needle aspiration for investigation of submucosal and extrinsic masses of the colon and rectum. Endoscopy 2005;37:154-160.

6. Amin K, Olyaee M, Tawfik O, Fan F. Endoscopic ultrasoundguided fine needle aspiration as a diagnostic and staging tool for rectal and perirectal lesions-an institutional experience. Ann Diagn Pathol 2013;17:494-497.

7. Nakai Y, Isayama H, Chang KJ, et al. Slow pull versus suction in endoscopic ultrasound-guided fine-needle aspiration of pancreatic solid masses. Dig Dis Sci 2014;59:1578-1585.

8. Levy MJ, Abu Dayyeh BK, Fujii LL, et al. Prospective evaluation of adverse events following lower gastrointestinal tract EUS FNA. Am J Gastroenterol 2014;109:676-685.

9. Holt B, Varadarajulu S. Endoscopic ultrasound-guided pelvic abscess drainage (with video). J Hepatobiliary Pancreat Sci 2015;22:12-15.

10. Weilert F, Shah JN, Marson FP, Binmoeller KF. EUS-guided coil and glue for bleeding rectal varix. Gastrointest Endosc 2012;76:915-916.

11. Moningi S, Walker AJ, Malayeri AA, et al. Analysis of fiducials implanted during EUS for patients with localized rectal cancer receiving high-dose rate endorectal brachytherapy. Gastrointest Endosc 2015;81:765-769. 